PoS $\quad \begin{aligned} & \text { PROCEEDINGS } \\ & \text { OF SCIENCE }\end{aligned}$

\title{
Reactor antineutrino detection using CHANDLER : A portable neutrino detector
}

\author{
Alireza Haghighat \\ Virginia Tech \\ E-mail: haghigha@vt.edu
}

Patrick Huber

Virginia Tech

E-mail: pahuberevt. edu

\section{Shengchao Li}

Virginia Tech

E-mail: scli@vt.edu

Jonathan M. Link

Virginia Tech

E-mail: jmlinkevt.edu

\section{Camillo Mariani}

Virginia Tech

E-mail: camillo@vt.edu

\section{Jaewon Park}

Virginia Tech

E-mail: jaewon.parkevt. edu

\section{Tulasi Subedi*}

Virginia Tech

E-mail: tpsubedi@vt.edu

The 20th International Workshop on Neutrinos (NuFact2018)

12-18 August 2018

Blacksburg, Virginia

${ }^{*}$ Speaker. 


\section{Introduction}

CHANDLER is a detector technology to detect antineutrinos from a nuclear reactor. It is made up of layers of wavelength shifting plastic scintillating cubes separated by the sheets of ${ }^{6} \mathrm{Li}$ loaded $\mathrm{ZnS}$ scintillator for neutron detection. An antineutrino from the reactor interacts with a proton in the detector producing a positron and a neutron; this process is known as inverse beta decay (IBD)

$$
\bar{v}_{e}+p \rightarrow e^{+}+n
$$

The emitted positron deposits its kinetic energy in the scintillator, giving a prompt signal which is followed by a delayed signal when the neutron captures on ${ }^{6} \mathrm{Li}$.

The MiniCHANDLER detector is an $80 \mathrm{~kg}$ prototype consisting of five layers of $8 \times 8$ cubes and 6 neutron sheets shown in Figure 1. The detector was installed inside our self contained Mobile Neutrino Lab and delivered to the North Anna Nuclear Power Plant where it took data from August 9, 2017 to November 2, 2017. During that period, we took about a third of reactor-off data and two third of reactor-on data.

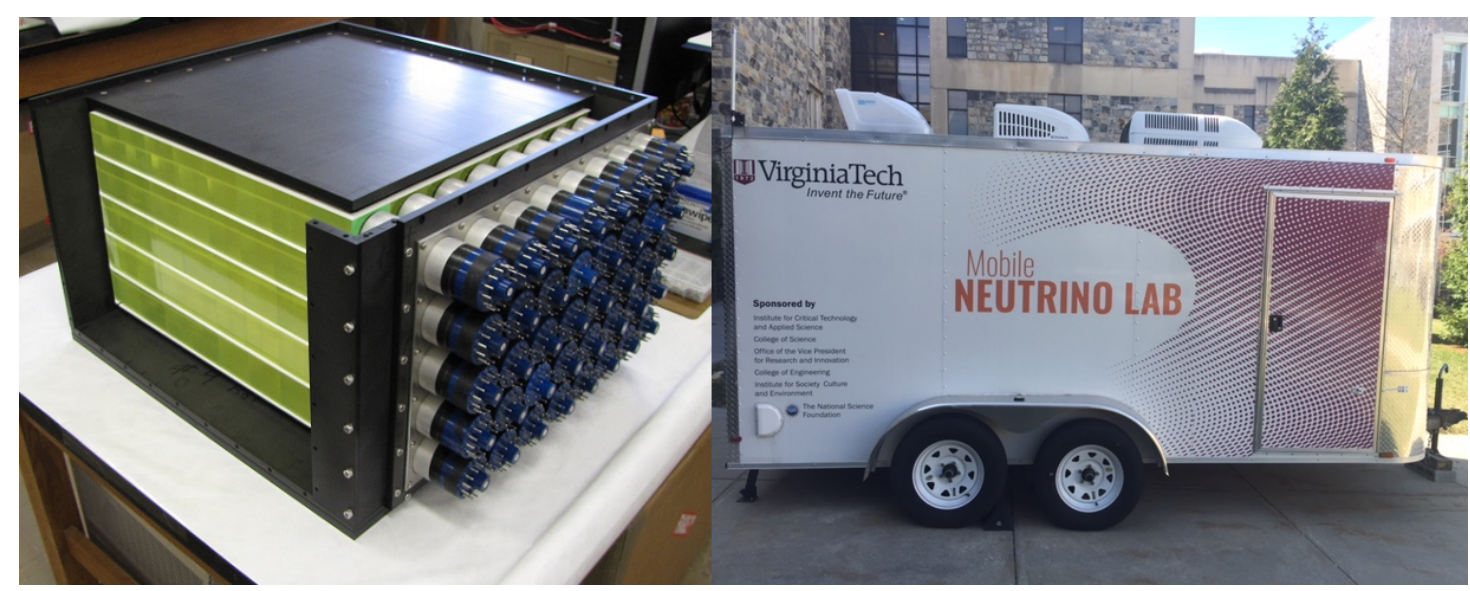

Figure 1: Left: the MiniCHANDLER detector during assembly, showing plastic cubes and neutron sheets, Right: the trailer in which MiniCHANDLER is installed

\section{IBD Analysis}

In the detector, both positron-like events (including $e^{+}$and gamma) and neutron captures make a pulse in the readout electronics. The positron-like events deposit energy in plastic scintillator, whereas the neutrons are captured in the sheet with $\mathrm{ZnS}$ scintillator. The short decay time of plastic scintillator gives a narrow pulse for positron-like events. However, the longer decay time of $\mathrm{ZnS}$ scintillator results in a broader pulse for neutrons. We can use these properties of the scintillators to discriminate the positron-like events from neutron captures.

We developed a template-based $\chi^{2}$-criterion to overcome the false neutron tag of PMT flashers and other electronics effects in naive neutron selection. In this method, the waveform is divided into eight regions and sum the ADC count over baseline in each region, normalized by total over all regions. Then, for the neutron template, we use 100 hand-selected neutron samples and calculate 
the mean of their normalized areas. Since, the positron-like pulse is contained entirely in the first region, the gamma template is trivial. We compare each waveform to the gamma and neutron templates to get their $\chi_{\gamma}^{2}$ and $\chi_{n}^{2}$. These two parameters in the $x$ and $y$-views are used to select the good neutron events.

To select IBD event candidates, we apply prompt/delayed spatial and temporal separation cuts. The 3D segmentation also allow us to apply topological cut to tag two $511 \mathrm{keV}$ positron annihilation gammas. We remove a large fraction of accidental backgrounds by fitting the prompt/delayed $\Delta t$ distribution of the IBD candidates. The accidental component has no $\Delta t$ dependence and appear flat in the distribution. Whereas, the correlated events which include the IBD events as well as the fast neutrons from cosmic rays, decay exponentially over time in the distribution.

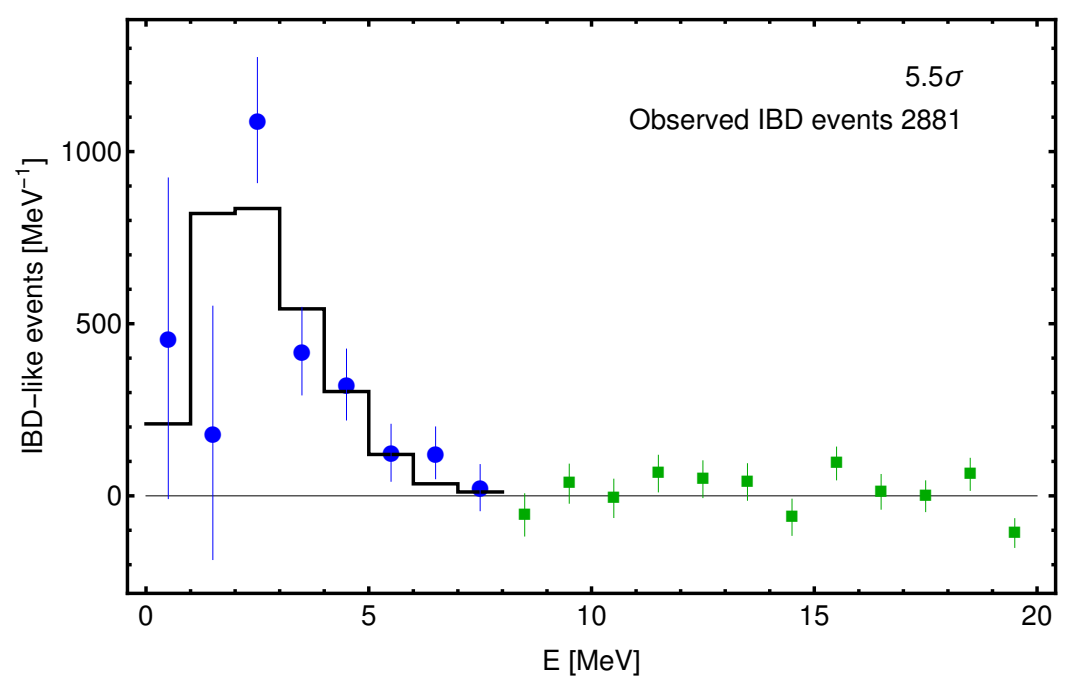

Figure 2: Result of reactor-on minus reactor-off correlated events. Green points show the normalization points and blue points show IBD signal with histogram showing Monte Carlo IBD spectrum

We calculate the number of correlated events in each $1 \mathrm{MeV}$ energy bin. Since, the IBD events from the reactor have energy less than $8 \mathrm{MeV}$, we scale the reactor-off events using the bins beyond $8 \mathrm{MeV}$ to match the reactor-on events. Then, we subtract reactor-off rates from reactor-on rates to get the IBD spectrum [1] as shown in Figure 2.

\section{Conclusion}

The subtracted event rate is consistent with expectations and the spectrum is consistent with our Monte Carlo generated IBD spectrum. This observation of reactor neutrinos make MiniCHANDLER the first street-legal mobile neutrino detector and the first, essentially unshielded detection of reactor neutrinos. This rapidly-deployable surface level detector technology could be used for nuclear non-proliferation and reactor monitoring applications.

\section{References}

[1] Haghighat, A., Huber, P., Li, S., Link, J. M., Mariani, C., Park, J., \& Subedi, T. (2018). Observation of Reactor Antineutrinos with a Rapidly-Deployable Surface-Level Detector. arXiv preprint arXiv:1812.02163 